\title{
Variation in the vertebral levels of the origins of the abdominal aorta branches: a retrospective imaging study
}

\author{
Jehad Fataftah ${ }^{1}$, Justin Z. Amarin ${ }^{2}$, Haya H. Suradi ${ }^{2}$, Maher T. Hadidi $^{3}$, Amjad T. Shatarat $^{4}$, \\ Abdel Rahman A. Al Manasra ${ }^{5}$, Samah Shahin ${ }^{6}$, Darwish H. Badran ${ }^{4}$ \\ ${ }^{1}$ Department of Internal and Family Medicine, Faculty of Medicine, The Hashemite University, Zarqa, ${ }^{2}$ School of Medicine, The University of Jordan, \\ Amman, ${ }^{3}$ Faculty of Medicine, Al-Balqa' Applied University, Al-Salt, ${ }^{4}$ Department of Anatomy and Histology, School of Medicine, The University \\ of Jordan, Amman, ${ }^{5}$ Department of General Surgery and Urology, Faculty of Medicine, Jordan University of Science and Technology, Irbid, ${ }^{6}$ Prince \\ Hamzah Hospital, Amman, Jordan
}

\begin{abstract}
Our aim was to investigate the variation in the vertebral levels of the origins of the celiac artery, superior and inferior mesenteric arteries, paired renal arteries, and common iliac arteries. We conducted a retrospective imaging study in a large public secondary hospital on a nonrandom sample of 227 participants. We consecutively included adult patients who had undergone computed tomography angiography of the abdomen and excluded patients with a history of any vertebral abnormality or whose images revealed evidence of a vertebral abnormality or a congenital anomaly of any of the branches of the abdominal aorta. The primary outcome was the frequency distribution of the vertebral levels of the landmarks. The secondary outcomes were the intercorrelations of the vertebral levels of the landmarks and their relationships with age, sex, weight, height, and body mass index. The celiac artery originated at T11/T12-L1/L2, followed by the superior mesenteric artery at T12-L2, the paired renal arteries at T12/L1-L2/L3, the inferior mesenteric artery at L2-L4, and the common iliac arteries at L3-L5. The vertebral levels of the landmarks were positively intercorrelated and stronger between proximate pairs. In addition, the vertebral levels of the landmarks were related to age, but not sex, weight, height, or body mass index. The intercorrelations suggest that a considerable proportion of the variation is accounted for by 'trickle-down' variation; variation in the vertebral level of a proximal landmark results in variation in the vertebral level of the immediate distal landmark. The overarching parameter remains unidentified.
\end{abstract}

Key words: Abdominal aorta, Anatomy, Anatomic variation, Anatomic landmarks, Computed tomography angiography

Received March 8, 2020; Revised June 2, 2020; Accepted June 8, 2020

\section{Introduction}

The aorta is the largest blood vessel in the human body.

\author{
Corresponding author: \\ Darwish H. Badran (10) \\ Department of Anatomy and Histology, School of Medicine, The \\ University of Jordan, Queen Rania Al-Abdullah Street, Amman 11942, \\ Jordan \\ E-mail: dhbadran@ju.edu.jo
}

It originates in the thorax and terminates in the abdomen, crossing from one cavity to the other through a hiatus in the diaphragm. The abdominal portion of the aorta, or the abdominal aorta, gives off anterior, lateral, posterior, and terminal branches: The anterior branches are the celiac, superior mesenteric, inferior mesenteric, and testicular or ovarian arteries; the lateral branches are the middle suprarenal, renal, and inferior phrenic arteries; the posterior branches are the lumbar and median sacral arteries; and, the terminal branches are the common iliac arteries [1].

Anatomists use the 33 vertebrae to reference the rela- 
tive position of internal landmarks. The vertebral levels of the origins of the abdominal aorta branches are especially convenient to measure because the abdominal aorta runs anterior to the vertebral column. Indeed, many authors have documented the vertebral levels of the landmarks, which vary between individuals. Many factors may explain the variation. The usual suspects are age, sex, ethnicity, weight, height, and body mass index [2-4]. However, some questions remain unanswered. The interrelationships of the vertebral levels of the landmarks are unclear, and the relationships between the factors and the vertebral levels of the landmarks are not well substantiated. Therefore, our aim was to investigate the variation in the vertebral levels of six landmarks: the origins of the celiac artery, superior and inferior mesenteric arteries, paired renal arteries, and common iliac arteries.

\section{Materials and Methods}

We conducted a retrospective imaging study in the Department of Radiology (Prince Hamzah Hospital) between January and March 2019. We drew our sample of participants from the picture archiving and imaging system of the department. We consecutively included adult patients $(\geq 18$ years of age) who had undergone computed tomography angiography of the abdomen using a 128-slice scanner. The images had been captured 15 seconds postinjection $(75 \mathrm{ml}$ iohexol $350[4 \mathrm{ml} / \mathrm{s}]$ and $50 \mathrm{ml}$ saline chaser $[5 \mathrm{ml} / \mathrm{s}])$. We excluded patients with a history of any vertebral abnormality or whose images revealed evidence of a vertebral abnormality or a congenital anomaly of the abdominal aorta branches. A member of our study team, who is a diagnostic radiologist with 10 years of experience, reviewed the computed tomography images of eligible patients and determined the vertebral levels of the origins of the celiac artery, superior and inferior mesenteric arteries, paired renal arteries, and common iliac arteries (Fig. 1). We also retrieved the age, sex, weight, and height of each patient from the system. We calculated the body mass index using the standard formula $\left(\mathrm{kg} \cdot \mathrm{m}^{-2}\right)$. The primary outcome was the frequency distribution of the vertebral levels of the landmarks. The secondary outcomes were the intercorrelations of the vertebral levels of the landmarks and their relationships with age, sex, weight, height, and body mass index. The study protocol was approved by the ethics committee of the Prince Hamzah Hospital (6/2/2019/2020).

\section{Statistical methods}

We used R (version 3.6.1; R Development Core Team, 2019; http://www.r-project.org) to perform power and data analyses. We calculated the minimum sample size for a test of correlation under the following assumptions: $\rho=0.3$, $\alpha=0.05, \beta=0.05$. We also calculated the minimum sample size for K. Pearson's $\chi^{2}$ test under the following assumptions: $\omega=0.3, d f=4, \alpha=0.05, \beta=0.05$. The minimum sample sizes were 138 and 207 participants, respectively, so the latter was our target. We summarized categorical data as $\mathrm{n}(\%)$ and continuous data as mean (SD). We calculated Kendall's $\tau_{\mathrm{B}}$ and the estimated $P$-value of the $\mathrm{z}$ test to measure the intercorrelations between the vertebral levels of the branch origins. To measure the uncertainty of each point estimate of Kendall's $\tau_{B}$, we computed the standard error of the statistic from 1,000 ordinary nonparametric bootstrap replicates, followed by the bias-corrected and accelerated confidence interval. We used the same to measure the correlations of the vertebral levels of the branch origins with age, weight, height, and body mass index. To measure the association of the vertebral levels of the branch origins with sex, we calculated K. Pearson's $\chi^{2}$. To account for some expected cell frequencies between one and five, we applied E. Pearson's $N-1$ correction to K. Pearson's $\chi^{2}$ and calculated the $P$-value of the corrected statistic [5]. To account for multiple testing, we corrected the $P$-values using the Benjamini-Hochberg procedure. We present numerical data according to the recommendations of Cole [6].

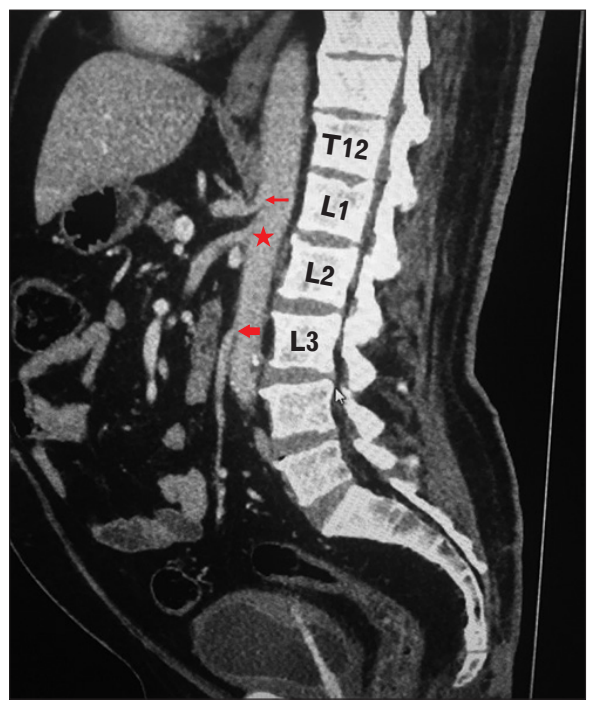

Fig. 1. Computed tomography angiography image showing the celiac (narrow red arrow), superior mesenteric (red star), and inferior mesenteric (wide red arrow) arteries. 


\section{Results}

We included 227 participants in the final analysis. No data were missing. The mean age, weight, height, and body mass index of the participants were $54 \pm 15$ years, $78 \pm 17 \mathrm{~kg}$, $166 \pm 8 \mathrm{~cm}$, and $28 \pm 6 \mathrm{~kg} \cdot \mathrm{m}^{-2}$ respectively. One hundred and six were female (46.7\%) and 121 were male (53.3\%).

We studied the vertebral levels of the origins of the celiac artery, superior and inferior mesenteric arteries, paired renal arteries, and common iliac arteries. The origins of all the branches varied between exactly two vertebral levels (Fig. 2).

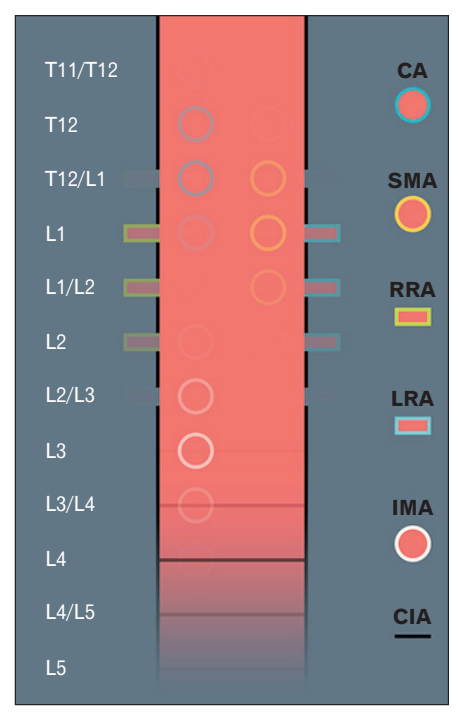

Fig. 2. The vertebral levels of the origins of the abdominal aorta branches ( $n=227$ participants). We used the proportions of incidence in our study to determine the degree of opacity of the visual representation of the variants. CA, celiac artery; CIA, common iliac arteries; IMA, inferior mesenteric artery; LRA, left renal artery; RRA, right renal artery; SMA, superior mesenteric artery.
The celiac artery originated at T11/T12-L1/L2, followed by the superior mesenteric artery at T12-L2, the paired renal arteries at T12/L1-L2/L3, the inferior mesenteric artery at L2-L4, and the common iliac arteries at L3-L5. We present the frequency distribution of the vertebral levels of the origins in Table 1.

The territories of the origins of the celiac and inferior mesenteric arteries were completely distinct; the origin of the celiac artery did not occur below L1/L2, and the origin of the inferior mesenteric did not occur above L2. The territories of the origins of the superior and inferior mesenteric arteries overlapped at L2 but were otherwise distinct. In relation to the left renal artery, the right renal artery originated at a higher level in 34 cases (15.0\%), the same level in 183 cases (80.6\%), and a lower level in 10 cases (4.4\%).

The vertebral levels of the origins of all branches were positively and statistically significantly intercorrelated. The intercorrelations were stronger between proximate branches than distant branches. We present the correlation matrix in Table 2. In addition, the vertebral levels of the origins of all branches were related to age, but not sex, weight, height, or body mass index (Table 3, Table 4).

\section{Discussion}

We conducted a retrospective imaging study of the variation in the vertebral levels of the origins of the abdominal aorta branches in a nonrandom sample of the Jordanian population. We found that the territories of all the landmarks spanned exactly two vertebral levels. In addition, we found that the vertebral levels of the landmarks were positively and statistically significantly intercorrelated and related to age,

Table 1. The vertebral levels of the origins of six abdominal aorta branches $(n=227)$

\begin{tabular}{|c|c|c|c|c|c|c|}
\hline Vertebral level & Celiac artery & $\begin{array}{c}\text { Superior mesenteric } \\
\text { artery }\end{array}$ & Right renal artery & Left renal artery & $\begin{array}{c}\text { Inferior mesenteric } \\
\text { artery }\end{array}$ & Common iliac arteries \\
\hline $\mathrm{T} 11 / \mathrm{T} 12$ & $10(4.4)$ & $0(0.0)$ & $0(0.0)$ & $0(0.0)$ & $0(0.0)$ & $0(0.0)$ \\
\hline $\mathrm{T} 12$ & $80(35.2)$ & $9(4.0)$ & $0(0.0)$ & $0(0.0)$ & $0(0.0)$ & $0(0.0)$ \\
\hline $\mathrm{T} 12 / \mathrm{L} 1$ & $96(42.3)$ & $69(30.4)$ & $9(4.0)$ & $3(1.3)$ & $0(0.0)$ & $0(0.0)$ \\
\hline $\mathrm{L} 1$ & $39(17.2)$ & $105(46.3)$ & $93(41.0)$ & $92(40.5)$ & $0(0.0)$ & $0(0.0)$ \\
\hline $\mathrm{L} 1 / \mathrm{L} 2$ & $2(0.9)$ & $41(18.1)$ & $82(36.6)$ & $78(34.4)$ & $0(0.0)$ & $0(0.0)$ \\
\hline L2 & $0(0.0)$ & $3(1.3)$ & $39(17.2)$ & $50(22.0)$ & $10(4.4)$ & $0(0.0)$ \\
\hline $\mathrm{L} 2 / \mathrm{L} 3$ & $0(0.0)$ & $0(0.0)$ & $4(1.8)$ & $4(1.8)$ & $58(25.6)$ & $0(0.0)$ \\
\hline L3 & $0(0.0)$ & $0(0.0)$ & $0(0.0)$ & $0(0.0)$ & $122(53.7)$ & $9(4.0)$ \\
\hline $\mathrm{L} 3 / \mathrm{L} 4$ & $0(0.0)$ & $0(0.0)$ & $0(0.0)$ & $0(0.0)$ & $32(14.1)$ & $40(17.6)$ \\
\hline $\mathrm{L} 4$ & $0(0.0)$ & $0(0.0)$ & $0(0.0)$ & $0(0.0)$ & $5(2.2)$ & $128(56.4)$ \\
\hline L4/L5 & $0(0.0)$ & $0(0.0)$ & $0(0.0)$ & $0(0.0)$ & $0(0.0)$ & $41(18.1)$ \\
\hline L5 & $0(0.0)$ & $0(0.0)$ & $0(0.0)$ & $0(0.0)$ & $0(0.0)$ & $9(4.0)$ \\
\hline
\end{tabular}

Data are presented as number (\%). 
Table 2. Intercorrelation of the vertebral levels of the origins of six abdominal aorta branches $(n=227)$

\begin{tabular}{lccccc}
\hline \multicolumn{1}{c}{ Artery } & Celiac artery & $\begin{array}{c}\text { Superior mesenteric } \\
\text { artery }\end{array}$ & Right renal artery & Left renal artery & $\begin{array}{c}\text { Inferior mesenteric } \\
\text { artery }\end{array}$ \\
\hline Superior mesenteric artery & $0.88(0.84-0.92) ;<0.001$ & & & & \\
Right renal artery & $0.65(0.57-0.73) ;<0.001$ & $0.67(0.59-0.74) ;<0.001$ & & & \\
Left renal artery & $0.67(0.59-0.73) ;<0.001$ & $0.68(0.59-0.74) ;<0.001$ & $0.82(0.76-0.87) ;<0.001$ & & \\
Inferior mesenteric artery & $0.44(0.34-0.53) ;<0.001$ & $0.50(0.41-0.58) ;<0.001$ & $0.52(0.42-0.59) ;<0.001$ & $0.51(0.40-0.58) ;<0.001$ & \\
Common iliac arteries & $0.41(0.31-0.51) ;<0.001$ & $0.46(0.37-0.53) ;<0.001$ & $0.47(0.38-0.55) ;<0.001$ & $0.44(0.34-0.52) ;<0.001$ & $0.68(0.59-0.75) ;<0.001$ \\
\hline
\end{tabular}

Data are Kendall's $\tau_{\mathrm{B}}(95 \%$ confidence interval); corrected $P$-value.

Table 3. Correlations of the vertebral levels of the origins of six abdominal aorta branches with age, weight, height, and body mass index ( $\mathrm{n}=227)$

\begin{tabular}{lcccccc}
\hline \multicolumn{1}{c}{ Measure } & Celiac artery & $\begin{array}{c}\text { Superior mesenteric } \\
\text { artery }\end{array}$ & Right renal artery & Left renal artery & $\begin{array}{c}\text { Inferior mesenteric } \\
\text { artery }\end{array}$ & $\begin{array}{c}\text { Common iliac } \\
\text { arteries }\end{array}$ \\
\hline Age & $0.21(0.11-0.31) ;$ & $0.22(0.11-0.31) ;$ & $0.27(0.17-0.37) ;$ & $0.26(0.15-0.35) ;$ & $0.27(0.17-0.36) ;$ & $0.26(0.16-0.35) ;$ \\
& $<0.001$ & $<0.001$ & $<0.001$ & $<0.001$ & $<0.001$ & $<.001$ \\
Weight & $0.03(-0.07$ to 0.14$) ;$ & $0.01(-0.10$ to 0.12$) ;$ & $0.03(-0.06$ to 0.14$) ;$ & $0.02(-0.08$ to 0.12$) ;$ & $0.06(-0.04$ to 0.17$) ; \quad 0.09(-0.02$ to 0.19$) ;$ \\
& 0.6 & 0.9 & 0.7 & 0.8 & 0.4 & 0.2 \\
Height & $0.09(-0.02$ to 0.20$) ;$ & $0.09(-0.02$ to 0.20$) ;$ & $0.07(-0.03$ to 0.17$) ;$ & $0.01(-0.10$ to 0.11$) ;$ & $-0.04(-0.16$ to 0.06$) ;$ & $0.04(-0.07$ to 0.14$) ;$ \\
& 0.2 & 0.2 & 0.3 & 0.9 & 0.6 & 0.6 \\
Body mass index & $0.01(-0.10$ to 0.12$) ;$ & $-0.01(-0.12$ to 0.09$) ;$ & $0.01(-0.09$ to 0.11$) ;$ & $0.02(-0.07$ to 0.12$) ;$ & $0.09(-0.03$ to 0.19$) ;$ & $0.07(-0.03$ to 0.17$) ;$ \\
& 0.9 & 0.9 & 0.9 & 0.8 & 0.2 & 0.3 \\
\hline
\end{tabular}

Data are Kendall's $\tau_{\mathrm{B}}(95 \%$ confidence interval); corrected $P$-value.

Table 4. Associations of the vertebral levels of the origins of six abdominal aorta branches with sex $(n=227)$

\begin{tabular}{lc}
\hline \multicolumn{1}{c}{ Branch } & $P$-value \\
\hline Celiac artery & 0.3 \\
\hline Superior mesenteric artery & 0.4 \\
\hline Right renal artery & 0.6 \\
\hline Left renal artery & 0.2 \\
\hline Inferior mesenteric artery & 0.6 \\
\hline Common iliac arteries & 0.6 \\
\hline
\end{tabular}

but not sex, weight, height, or body mass index.

The vertebral levels of the origins of the abdominal aorta branches have long since been reported in cadaveric studies of samples drawn from diverse populations. Our results are consistent with seminal reports from Canada, Japan, and Germany [7]. Recent imaging studies, based in India and Greece, have also reported similar results $[2,8]$. Therefore, ethnicity does not appear to be a significant contributor to the variation in the vertebral levels of the landmarks. We went further and measured the intercorrelations between the vertebral levels of the origins. Our findings suggest that variation in the most proximal branch of the abdominal aorta 'trickles down' to the distal branches. However, 'trickle-down' variation explained a part, but not all of the variation.

We studied the relationships of the variation with age, sex, weight, height, and body mass index. Separate authors have long since reported a distal shift of abdominal structures, including the branches of the abdominal aorta, with age [7]. The whole length of the aorta increases with age because elastin fibers are replaced with collagen fibers [9]. Our results are consistent with the foregoing references; we found consistent correlations between age and the vertebral levels of the origins. In contrast, three teams of authors recently reproduced the correlation for some arteries but not others, or none at all [2-4]. The sample sizes in all three studies were less than half of our sample size, so differences in power may explain the inconsistency. In addition, we found no association between sex and the variation, which is consistent with previous reports [2-4]. Finally, one team reported a relationship between body mass index and the vertebral level of the origin of the celiac artery but not the other arteries [3]. In contrast, we found no correlation between weight, height, and body mass index and the vertebral level of the origin of any branch.

Our study design limits the interpretability of our results. First, our study was not longitudinal, so we cannot substantiate the cause of the correlations between age and the vertebral levels of the origins of the abdominal aorta branches. The correlations may result from generational variation due to a factor or a set of factors we did not consider. Second, we used a nonrandom sampling technique, so our estimates likely do not represent the Jordanian population. Third, our study was underpowered to detect weak relationships, so 
our results must be interpreted with caution. Nevertheless, the power of our study is higher than the other studies referenced in our report. In addition, we show that a considerable part of the variation in the vertebral levels of the origins is due to intercorrelations, which is a new finding with possible implications. For example, variation in the position of a yet unidentified proximal landmark may explain the variation in the vertebral levels of the origins of the abdominal aorta branches ('trickle-down' variation).

In conclusion, the intercorrelations suggest that a considerable proportion of the variation in the vertebral levels of the origins of the abdominal aorta branches is accounted for by 'trickle-down' variation; variation in the vertebral level of a proximal landmark results in variation in the vertebral level of the immediate distal landmark. The overarching parameter remains unidentified.

\section{ORCID}

Jehad Fataftah: https://orcid.org/0000-0003-0812-5695

Justin Z. Amarin: https://orcid.org/0000-0002-4484-1077

Haya H. Suradi: https://orcid.org/0000-0003-3893-9294

Maher T. Hadidi: https://orcid.org/0000-0003-3801-5470

Amjad T. Shatarat: https://orcid.org/0000-0002-2101-0174

Abdel Rahman A. Al Manasra:

https://orcid.org/0000-0003-4556-6000

Samah Shahin: https://orcid.org/0000-0002-7291-2717

Darwish H. Badran:

https://orcid.org/0000-0002-8117-1762

\section{Author Contributions}

Conceptualization: JF, MTH, ATS, DHB. Data acquisition: JF, AAA, SS, DHB. Data analysis or interpretation: JZA,
HHS. Drafting of the manuscript: JZA, HHS. Critical revision of the manuscript: all authors. Approval of the final version of the manuscript: all authors.

\section{Conflicts of Interest}

No potential conflict of interest relevant to this article was reported.

\section{References}

1. Drake RL, Vogl AW, Mitchell AWM. Gray's anatomy for students. 2nd ed. Edinburgh: Churchill Livingstone; 2009.

2. Goyal R, Aggarwal A, Gupta T, Gulati A, Jaggi S, Mirjalili SA, Sahni D. Reappraisal of the classical abdominal anatomical landmarks using in vivo computerized tomography imaging. Surg Radiol Anat 2020;42:417-28.

3. Mirjalili SA, McFadden SL, Buckenham T, Stringer MD. A reappraisal of adult abdominal surface anatomy. Clin Anat 2012;25:844-50.

4. Shen XH, Su BY, Liu JJ, Zhang GM, Xue HD, Jin ZY, Mirjalili SA, Ma C. A reappraisal of adult thoracic and abdominal surface anatomy via CT scan in Chinese population. Clin Anat 2016;29:165-74.

5. Campbell I. Chi-squared and Fisher-Irwin tests of two-bytwo tables with small sample recommendations. Stat Med 2007;26:3661-75.

6. Cole TJ. Too many digits: the presentation of numerical data. Arch Dis Child 2015;100:608-9.

7. George R. Topography of the unpaired visceral branches of the abdominal aorta. J Anat 1935;69(Pt 2):196-205.

8. Panagouli E, Lolis E, Venieratos D. A morphometric study concerning the branching points of the main arteries in humans: relationships and correlations. Ann Anat 2011;193:86-99.

9. Komutrattananont P, Mahakkanukrauh P, Das S. Morphology of the human aorta and age-related changes: anatomical facts. Anat Cell Biol 2019;52:109-14. 